\title{
Formation of wall-less cells in Kitasatospora viridifaciens requires cytoskeletal protein FilP in oxygen-limiting conditions
}

\author{
Eveline Ultee $^{1,2} \mid$ Xiaobo Zhong $^{1,2}$ | Shraddha Shitut ${ }^{1,2}$ | Ariane Briegel (1D) ${ }^{1,2}$ | \\ Dennis Claessen (1D) ${ }^{1,2}$
}

${ }^{1}$ Department of Molecular Biotechnology, Institute of Biology, Leiden University, Leiden, The Netherlands

${ }^{2}$ Centre for Microbial Cell Biology, Leiden University, Leiden, The Netherlands

\section{Correspondence}

Dennis Claessen, Department of Molecular Biotechnology, Institute of Biology, Leiden University, Sylviusweg 72, 2333 BE Leiden, The Netherlands.

Email: d.claessen@biology.leidenuniv.nl

\section{Funding information}

Horizon2020 programme of the European Commission, Grant/Award Number: PID:2265; Vici grant from NWO, Grant/ Award Number: VI.C.192.002

\begin{abstract}
The cell wall is considered an essential component for bacterial survival, providing structural support, and protection from environmental insults. Under normal growth conditions, filamentous actinobacteria insert new cell wall material at the hyphal tips regulated by the coordinated activity of cytoskeletal proteins and cell wall biosynthetic enzymes. Despite the importance of the cell wall, some filamentous actinobacteria can produce wall-deficient S-cells upon prolonged exposure to hyperosmotic stress. Here, we performed cryo-electron tomography and live cell imaging to further characterize S-cell extrusion in Kitasatospora viridifaciens. We show that exposure to hyperosmotic stress leads to DNA compaction, membrane and S-cell extrusion, and thinning of the cell wall at hyphal tips. Additionally, we find that the extrusion of S-cells is abolished in a cytoskeletal mutant strain that lacks the intermediate filament-like protein FilP. Furthermore, micro-aerobic culturing promotes the formation of S-cells in the wild type, but the limited oxygen still impedes S-cell formation in the $\Delta$ filP mutant. These results demonstrate that S-cell formation is stimulated by oxygen-limiting conditions and dependent on functional cytoskeleton remodeling.

KEYWORDS

cell wall-deficiency, filamentous actinomycetes, FilP, micro-aerobic growth, osmotic stress
\end{abstract}

\section{1 | INTRODUCTION}

Almost all bacteria are enveloped by a cell wall that provides protection against challenging or hostile environments. The cell wall also prevents cells from bursting by withstanding the high internal turgor pressure. Furthermore, it is a major determinant of bacterial cell shape. A main constituent of the cell wall is peptidoglycan (PG), which consists of cross-linked glycan strands that create in essence a single gigantic molecule called the sacculus (Höltje, 1998). The biosynthesis of PG is conserved in bacteria and is, therefore, a prominent target for many clinically used antibiotics. Cell wall precursors are synthesized in the cytosol and are subsequently transferred to the exterior of the cell. Here, designated synthases incorporate these monomers into the preexisting cell wall (Pazos et al., 2017; van Teeffelen and Renner, 2018; Typas et al., 2012).

The site of incorporation of new cell wall material is species-specific and occurs over the entire surface of the lateral wall in many rod-shaped bacteria, while incorporation is confined to apical sites in filamentous actinobacteria. Tip growth in filamentous actinobacteria is orchestrated by the TIPOC (tip organizing center) multiprotein complex (Flärdh et al., 2012; Fuchino et al., 2013; Hempel et al., 2008; Holmes et al., 2013). The best-studied component of

This is an open access article under the terms of the Creative Commons Attribution-NonCommercial-NoDerivs License, which permits use and distribution in any medium, provided the original work is properly cited, the use is non-commercial and no modifications or adaptations are made.

(c) 2020 The Authors. Molecular Microbiology published by John Wiley \& Sons Ltd 

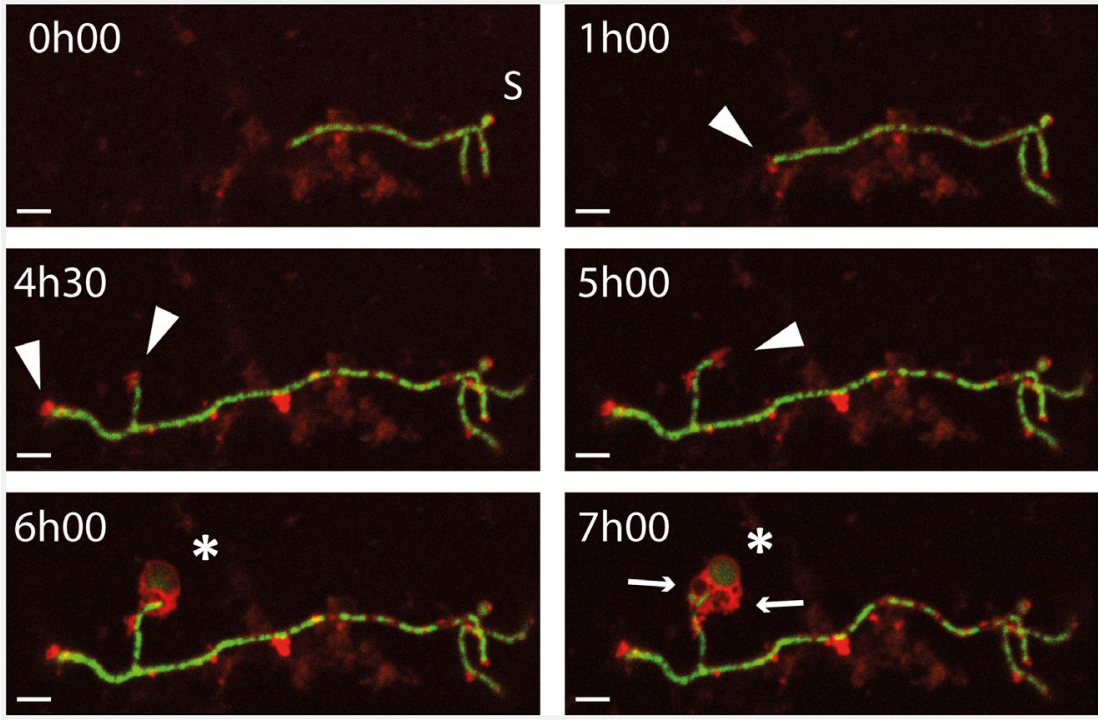

FIGURE 1 Extrusion of S-cells from $K$. viridifaciens germlings under high osmotic stress. Germinated K. viridifaciens spores were fluorescently labeled with SYTO9 (nucleic acids) and FM5-95 (lipids) and were grown under high osmotic conditions. Micrographs were taken every 30 min (see Supplementary Movie 1) of which a selection of micrographs are shown. The "S" indicates the spore, the arrowheads highlight the blebbing of membrane, the asterisk indicates the $\mathrm{S}$-cell, and the arrows show the formation of membrane vesicles. Scale bar: $5 \mu \mathrm{m}$ this complex is the essential protein DivIVA, which drives apical growth by recruiting the cell wall synthesis machinery and marks the location of new hyphal branch sites (Flärdh, 2003; Flärdh et al., 2012; Hempel et al., 2008). In order for the cell wall to grow, the existing PG needs to be modified to allow new monomers to be incorporated and cross-linked into the sacculus, making the hyphal tip a putative vulnerable structure. Previously, the intermediate filament-like protein FilP was proposed to reinforce the tip intracellularly (Bagchi et al., 2008). FilP localizes to active growing hyphal tips, where it forms cytoskeletal structures that interact with DivIVA (Fuchino et al., 2013). Unlike divIVA, filP is not essential and the absence of this gene in Streptomyces coelicolor causes a defect in growth and the morphology of the mycelium (Bagchi et al., 2008).

Although the cell wall is considered an essential structure in bacteria, many species can shed their cell wall to overcome PGtargeting threats, such as antibiotics and the mammalian immune system (Claessen and Errington, 2019; Dörr et al., 2016; Kawai et al., 2018; Monahan et al., 2014). In laboratory conditions, the transition from a walled state to the cell wall-deficient (CWD) state is typically induced by exposing bacteria to PG synthesis-targeting antibiotics and/or lytic enzymes, yielding so-called L-forms (Allan et al., 2009; Leaver et al., 2009). Our lab has previously shown that several filamentous actinobacteria have a natural ability to form CWD cells without the help of PG synthesis-targeting compounds (Ramijan et al., 2018). These CWD cells, termed S-cells for stress-induced cells, are extruded from hyphal tips in hyperosmotic environments following an arrest in tip growth. Compared to L-forms, S-cells are typically larger in size and unable to proliferate without their cell wall (Ramijan et al., 2018). Notably, S-cells can sustain in their CWD state for prolonged periods of time before switching to the canonical filamentous mode-of-growth (Ramijan et al., 2018). How S-cells are extruded and how this process is regulated at the molecular level is poorly understood.

In this study, we combine genetics with fluorescence time-lapse microscopy and cryo-electron tomography (cryo-ET) to characterize the morphological and structural changes associated with S-cell formation. Our data reveal that oxygen limitation triggers S-cell formation in the wild-type strain in a FilP-dependent manner. These results suggest that $\mathrm{S}$-cell extrusion is a controlled physiological adaptation to stress and depends on cytoskeletal elements involved in polar growth.

\section{2 | RESULTS}

\subsection{Membrane and DNA organization during S-cell extrusion}

We previously showed that prolonged exposure to hyperosmotic stress causes an increase in branching frequency, membrane synthesis, and DNA condensation in K. viridifaciens (Ramijan et al., 2018). To characterize these changes in more detail, we performed time-lapse microscopy in combination with fluorescent dyes that bind to nucleic acids and lipids (SYTO9 and FM5-95, respectively). Time-lapse imaging of growing filaments indeed revealed condensed DNA and an excess of membrane in high osmotic conditions (Supplementary Movies 1A, B). Strikingly, excess membrane was frequently extruded from the hyphal tips of both leading tips and emerging branches (Supplementary Movie 1A, Figure 1, arrowheads). Regrowth of the hyphal tip is associated with strong turns or bends, which could indicate a local rearrangement of the TIPOC leading to a new growth direction (Supplementary Movie 1B). In some cases, the membrane that blebs off from the hyphal tip enlarges and forms large vesicles with a diameter of 4-5 $\mu \mathrm{m}$ (Figure 1, asterisk in $6 \mathrm{h00}$ panel). Some of these vesicles emit green fluorescence, indicating the presence of SYTO9-stained nucleic acids, and therefore, we consider them S-cells. Subsequently, extruded smaller vesicles at the same tip are typically smaller and often lack nucleic acids (Figure 1, arrows in 7h00 panel). The hyphae still possess DNA after extruding S-cells. This could indicate that either DNA replication is ongoing, or that the nucleoid is changing its organization and morphology upon exposure to stress. 


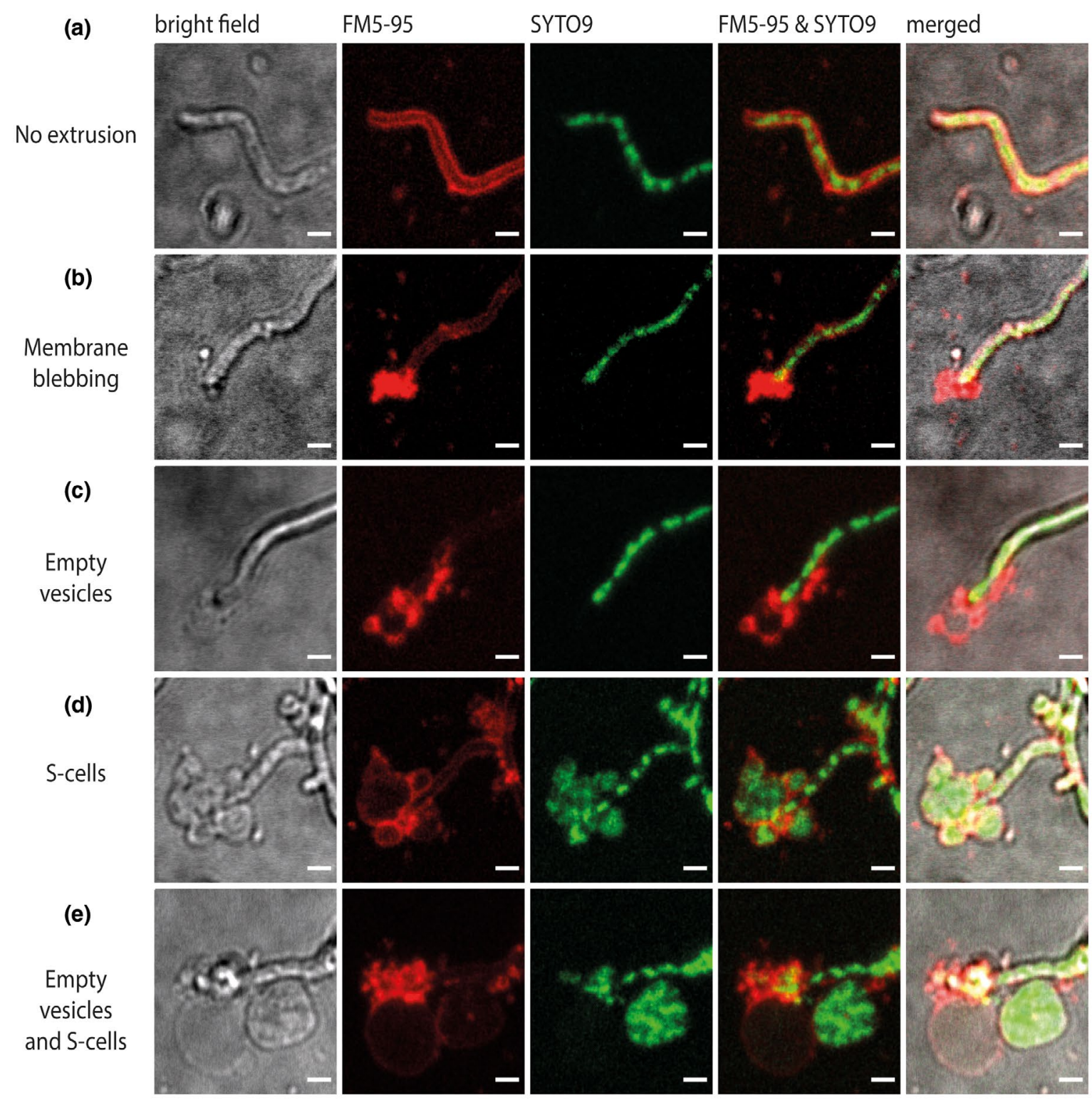

Quantification of S-cell related stress response events

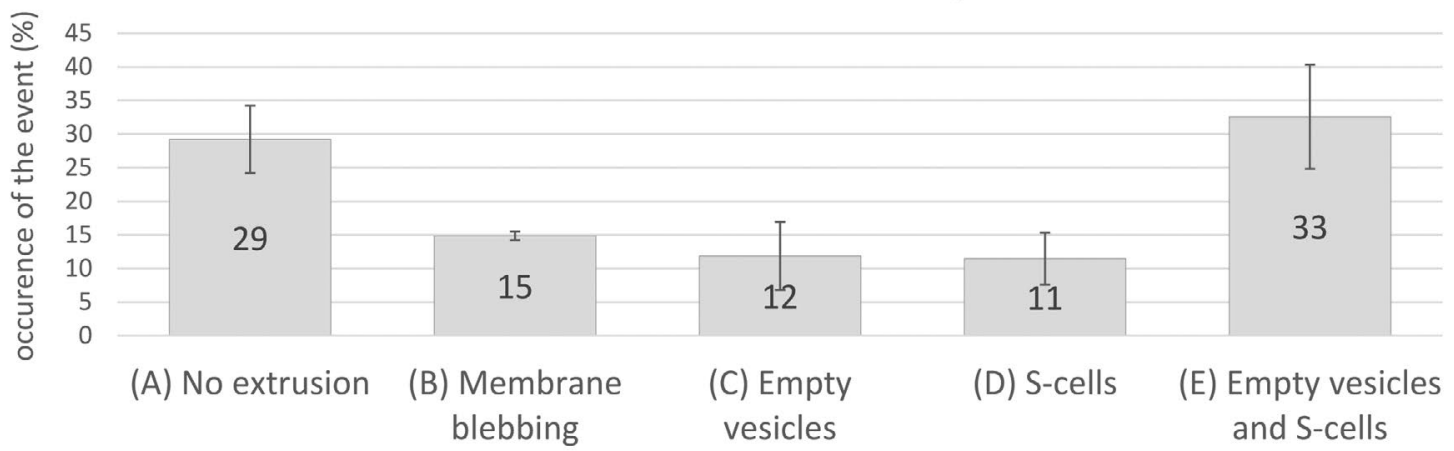

FIGURE 2 Quantification of S-cell-related stress response events at the hyphal tip. Germinated K. viridifaciens spores were fluorescently labeled with SYTO9 (nucleic acids) and FM5-95 (lipids) and were grown under high osmotic conditions. After 18 hr of growth, the hyphal tips were imaged (representative micrographs shown), examined, and categorized into (a) no visible S-cell-related stress responses, (b) membrane blebbing tips, (c) the presence of empty vesicles, (d) the presence of S-cells, or (e) the presence of empty vesicles and S-cells. The average of three experimental replicas is shown in the graph (data in Supplementary Table 1). Scale bars represent $2 \mu \mathrm{m}$ 
To quantify these observations, we categorized and quantified the various events associated with the formation and release of S-cells. In order of appearance, these stress response-related events are: (B) membrane extrusion without the formation of obvious membrane vesicles, (C) formation of empty membrane vesicles, (D) formation of DNA-containing S-cells, and (E) the presence of both S-cells and empty membrane vesicles (Figure 2). Quantitative analysis shows that after 18 hours of exposure to osmotic stress, on average $71 \%$ of the hyphal tips showed one of the abovementioned S-cell-related stress responses (responses B-D), while $29 \%$ of the tips appeared not affected, perhaps suggesting a stochastic effect (A). About $15 \%$ of all imaged and analyzed tips showed excessive membrane blebbing, while DNA-containing S-cells were observed surrounding $44 \%$ of the hyphal tips (Figure 2 , Supplementary Table 1). The majority of tips surrounded by S-cells also showed empty vesicles and/or excessive membrane blebbing. Of three experimental replicas, the number of non-responding and blebbing hyphal tips shows relatively little variance, whereas the occurrence of empty and/or filled vesicles differs per replica (Supplementary Table 1).

\subsection{Structural changes in the cell envelope accompany S-cell extrusion}

In order to observe the structural changes in the hyphal tip during $\mathrm{S}$-cell release, cryo-transmission electron microscopy (cryo-TEM) was used (Figure 3a,b). Interestingly, the cell envelope thickness, measured from the cytoplasmic membrane to the outer edge of the cell wall, was considerably thinner when $K$. viridifaciens was grown in medium supplemented with $20 \%$ sucrose: $31 \mathrm{~nm}( \pm 1,14 ; n=15)$, compared to an average of $43 \mathrm{~nm}( \pm 1,05 ; n=15)$ when grown in the same medium without sucrose (Supplementary Table 2).

Osmotically stressed $K$. viridifaciens cells were further investigated with cryo-electron tomography (cryo-ET), allowing three-dimensional visualization of the filaments and S-cells at high resolution. Despite the thickness of the hyphal cells for penetration of the electrons (>500 nm) and used buffer (20\% sucrose, resulting in low contrast), we were able to detect structural elements. Ribosomes can be distinguished inside the hyphal cells but are absent in the extreme end of the tip (Figure 3b). In fact, roughly 200-400 nm of the tip's apex does not appear to contain ribosomes or DNA. Fluorescent labeling of the chromosome in both stressed and non-stressed filaments confirm the absence of the chromosome at the extreme end of the tip (Figure 3c), which is in line with earlier findings in S. coelicolor (Kois-Ostrowska et al., 2016). We also analyzed extruded S-cells and membrane vesicles with cryo-ET. In line with the light microscopy data, the released S-cells ranged in size and content. While some vesicles contain ribosomes, DNA, and/or inner vesicles (Figure 4a,b), others appear empty (Figure 4c). Notably, all imaged S-cells lacked a visible cell wall, and were comparable to cells of a penicillin-induced L-form strain of K. viridifaciens (Ramijan et al., 2018) (Figure 4d). (a)
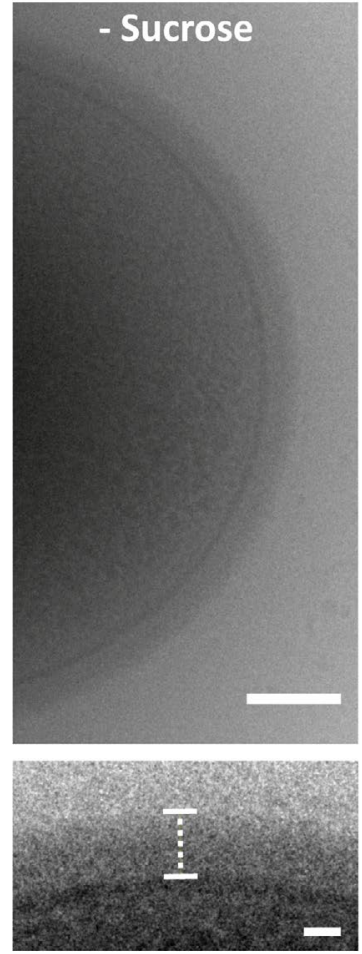
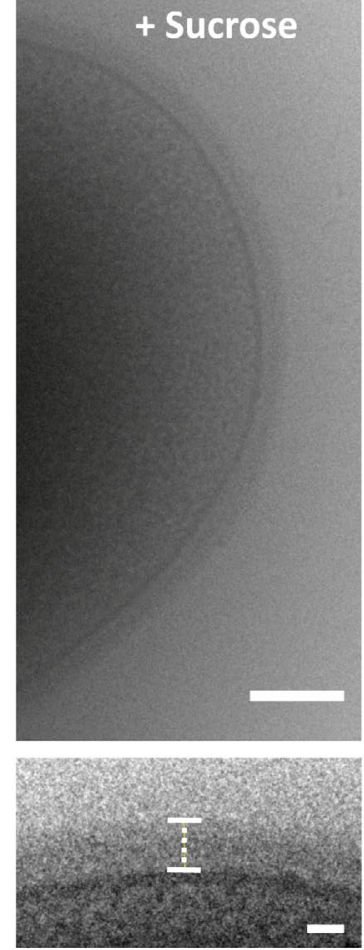

(b)

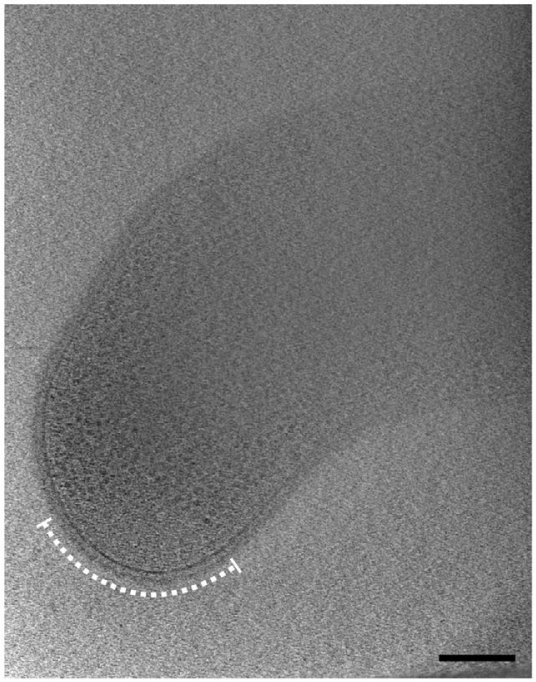

(c)
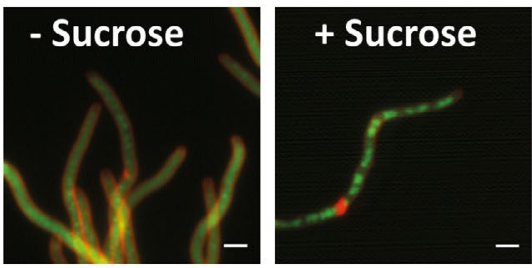

FIGURE 3 Structural changes in K. viridifaciens hyphae upon exposure to osmotic stress. Cryo-TEM micrographs of the tip of $K$. viridifaciens hyphae grown with or without $20 \%$ sucrose show a difference in cell wall thickness, highlighted in the insets (a). Cryo-ET micrograph of a 17-nm thick slice of $K$. viridifaciens grown with osmotic stress shows a ribosome excluded zone at the terminus of the tip (b). Fluorescently stained K. viridifaciens hyphae (lipids: FM5-95, red) show absence of nucleic acids at the tip and condensation of the DNA (SYTO9, green) upon exposure to osmotic stress (c). Scale bars represent $100 \mathrm{~nm}$ (upper panels A), $20 \mathrm{~nm}$ (lower panels A), $200 \mathrm{~nm}$ (B), or $5 \mu \mathrm{m}(\mathrm{C})$ 

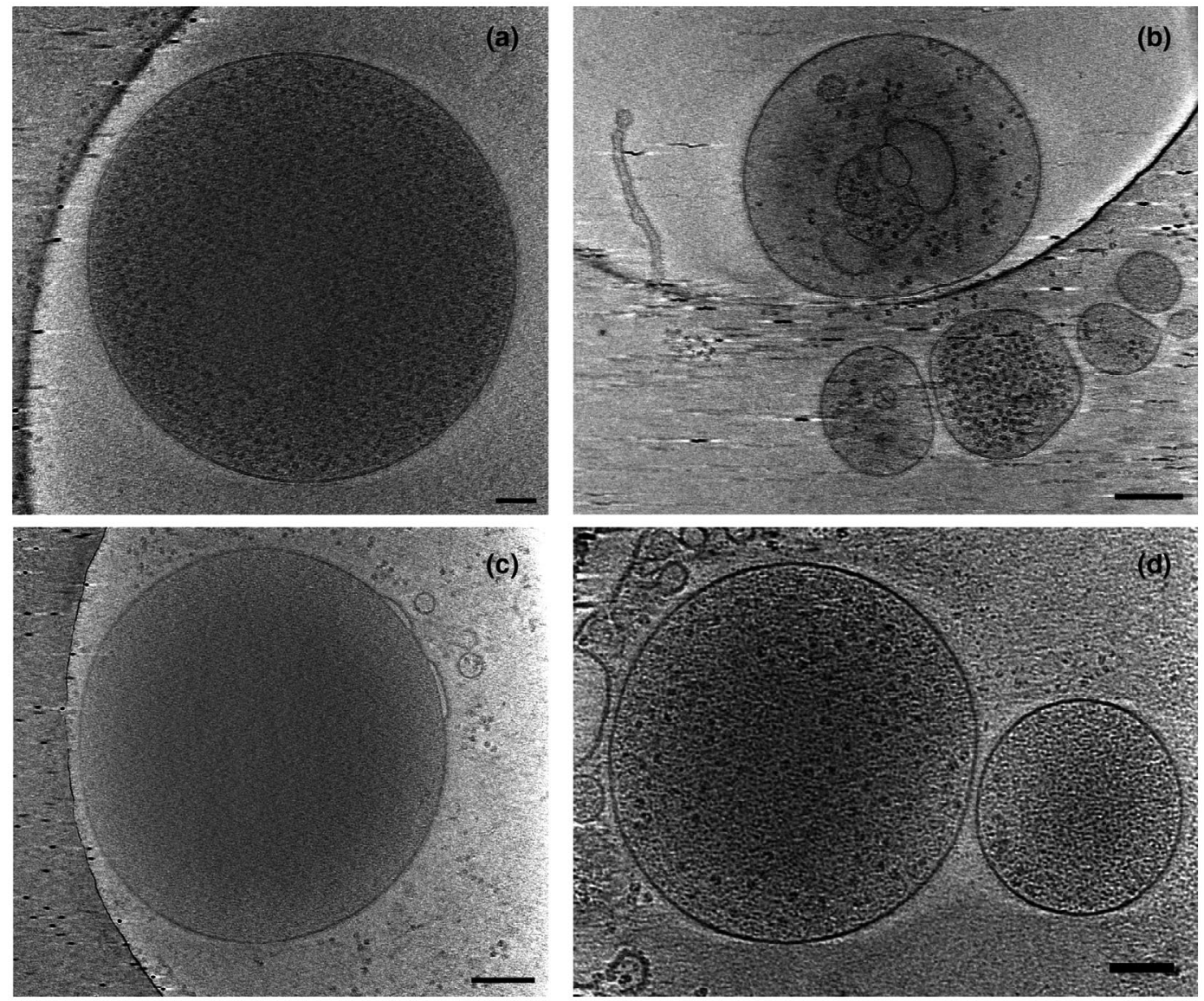

FIGURE 4 Heterogeneity among K. viridifaciens S-cells. Cryo-ET micrographs show S-cells formed by young hyphae of K. viridifaciens after 6 hours of exposure to high sucrose levels $(a-c)$. The S-cells structurally resemble L-forms (d). Scale bars represent $100 \mathrm{~nm}$ (panels $A$ and D) or $200 \mathrm{~nm}$ (panels B and C)

\subsection{The presence of FilP is required for the formation of S-cells}

Our current and previous data show that the hyphal tip undergoes a visible structural transition prior to S-cell release, which itself coincides with an arrest in tip growth (Ramijan et al., 2018). Since the cytoskeletal protein FilP localizes to actively growing hyphal tips and coordinates tip growth in conjunction with DivIVA (Bagchi et al., 2008), we asked whether it could play a role in the release of S-cells from osmotically stressed hyphae. We therefore created a knockout mutant strain of the filP homolog in K. viridifaciens (BOQ63_RS19300 (Ramijan et al., 2017), Supplementary Figure 1), which we subsequently also complemented in trans. In the absence of hyperosmotic stress conditions (MYM), the colonies of the $\Delta$ filP mutant were smaller than those of the wild-type strain and the complemented mutant (Supplementary Figure 2), consistent with a more compact manner of growth (see also Supplementary Movies 2-4). Despite the difference in colony size, all strains sporulated well after 7 days. These data are in line with previous observations in S. coelicolor (Bagchi et al., 2008). When the strains were exposed to hyperosmotic stress, the wild-type and complemented $\Delta$ filP mutant were able to extrude S-cells (Figure 5, Supplementary Movies 5, 7) while the $\Delta$ filP strain was not (Supplementary Movie 6). Instead, the mycelium of this mutant started to form numerous small side branches and adopted a highly compact morphology (Figure 5, Supplementary Movie 6). Further investigation using fluorescent dyes showed that not only the formation of S-cells was impaired in the $\Delta$ filP mutant, but also the extrusion of excess membrane (Figure $5 b$ ). This contrasts with the wildtype and complemented $\Delta$ filP mutant, which produce an abundance of S-cells, vesicles, and extruded membrane (Figure 5b). Interestingly, the morphology of the $\Delta$ filP mutant was comparable to the wild type when the strain was grown on LPMA plates without being covered by an agar pad (Supplementary Figure 3). Under these conditions, the $\Delta$ filP mutant was able to generate S-cells, but again failed to do so when an agar pad was placed on top of the growing mycelium (Supplementary Figure 3). The $\Delta$ filP mutant was also able to extrude S-cells when grown under slow shaking conditions in liquid LPB medium (Supplementary Figure 4). Altogether, these data show that the presence of FilP is important for S-cell formation and that the inability of the $\Delta$ filP mutant to generate $\mathrm{S}$-cells is not exclusively related to hyperosmotic stress.

\section{4 | Oxygen-limiting conditions stimulate S-cell formation in a FilP-dependent manner}

Our results indicated that FilP is important for the formation of $\mathrm{S}$-cells in conditions when the mycelium was grown in a confined 
(a)
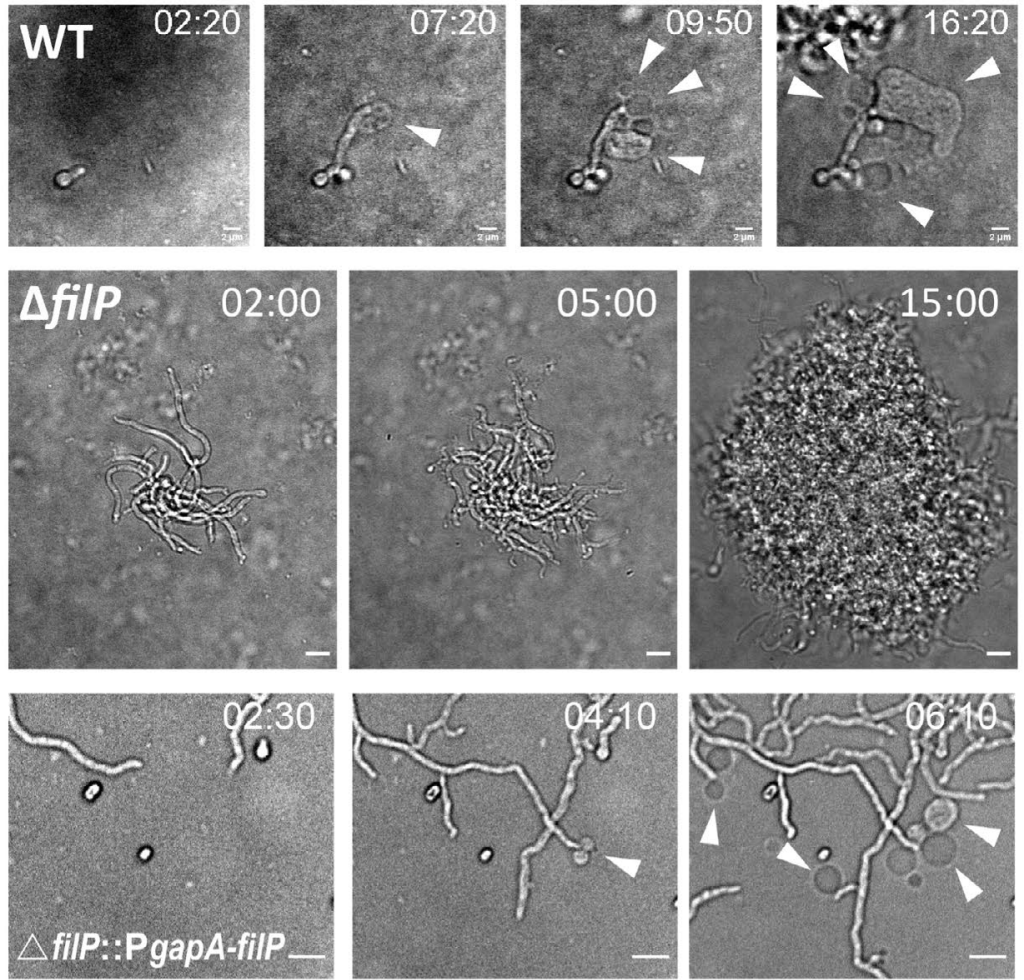

Brightfield

(b)
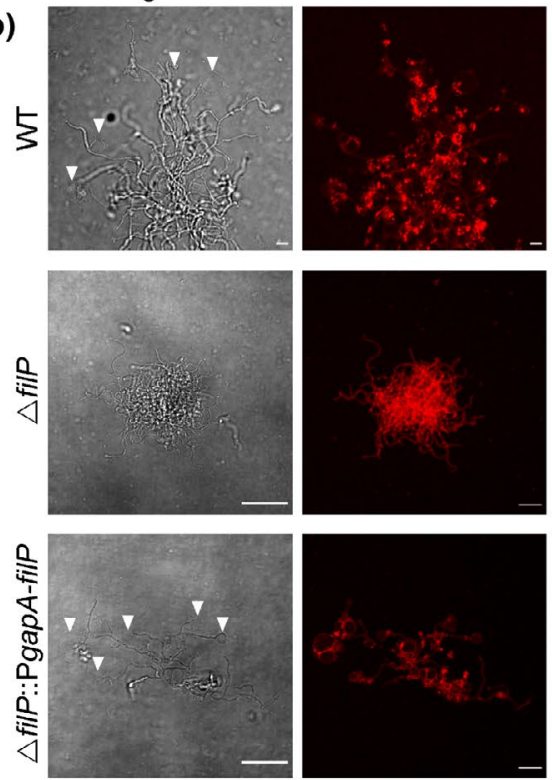
SYTO 9
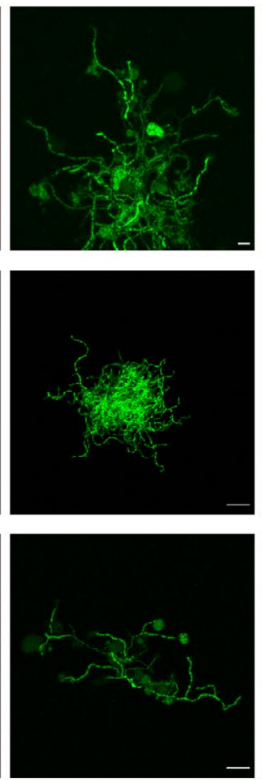
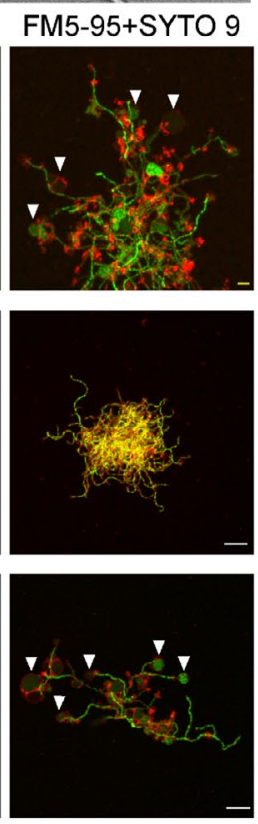

FIGURE 5 S-cell extrusion in $K$. viridifaciens depends on the presence of FilP. (a) Time-lapse microscopy stills of $K$. viridifaciens germlings under LPMA agar pads. Wild-type germlings (top panels) show S-cell formation, whereas those of the $\Delta$ filP strain do not form S-cells (middle panels). Instead, the $\Delta$ filP strain hyper-branches leading to the formation of dense mycelial pellets. Reintroduction of the filP gene in the $\Delta$ filP strain restored the ability to form S-cells (bottom panels). Stills were taken from Supplementary Movies 5-7 representing the wild-type, $\Delta$ filP mutant, and the complemented $\Delta$ filP strain, respectively. (B) Micrographs of mycelia of the $K$. viridifaciens wildtype (top), $\Delta$ filP mutant (middle), and complemented $\Delta$ filP mutant strain (bottom) that were fluorescently labeled with SYTO9 (nucleic acids, green) and FM5-95 (lipids, red) and grown underneath an LPMA agar pad. Images were taken after $18 \mathrm{hr}$ of growth. Arrows highlight the formation of S-cells. Time is indicated in the hours:minutes format and scale bars represent $2 \mu \mathrm{m}$ (A) or $5 \mu \mathrm{m}$ (B) space in between two layers of agar. We reasoned that this setup could potentially reduce the levels of oxygen that is accessible to the cells. To further study the effect of oxygen limitation on S-cell formation, the $K$. viridifaciens wild-type strain, the $\Delta$ filP mutant and the complemented $\Delta$ filP mutant strain were grown on solid LPMA and MYM medium in micro-aerobic vessels. All strains grew slowly in micro-aerobic conditions but were able to germinate and establish a mycelium after 14 days of growth (Figure 6). On MYM medium, the wild-type strain and the complemented $\Delta$ filP mutant formed a regular-looking mycelium consisting of branching hyphae. In some instances, apical branching or tip splitting could be observed (Figure 6, asterisks) which indicates a distortion in the TIPOC functioning
(Holmes et al., 2013). In contrast, the $\Delta$ filP mutant did not show obvious tip splitting, and formed short and apparently thick mycelial fragments that appear to have a lower branching frequency (Figure 6). Importantly, when cultivated micro-aerobically and exposed to osmotic stress, the wild-type strain and the complemented $\Delta$ filP mutant strain formed an excessive number of S-cells and vesicles that varied in size. Moreover, wild-type hyphal tips often showed signs of bulging reminiscent of sporulation (inlays in Figure 6). Bulging of hypha was also observed in the $\Delta$ filP strain, but the mutant strain was unable to form S-cells under these micro-aerobic conditions. Again, reintroduction of the filP gene restored the ability to form S-cells in the $\Delta$ filP mutant (Figure 6). Altogether, these data indicate 

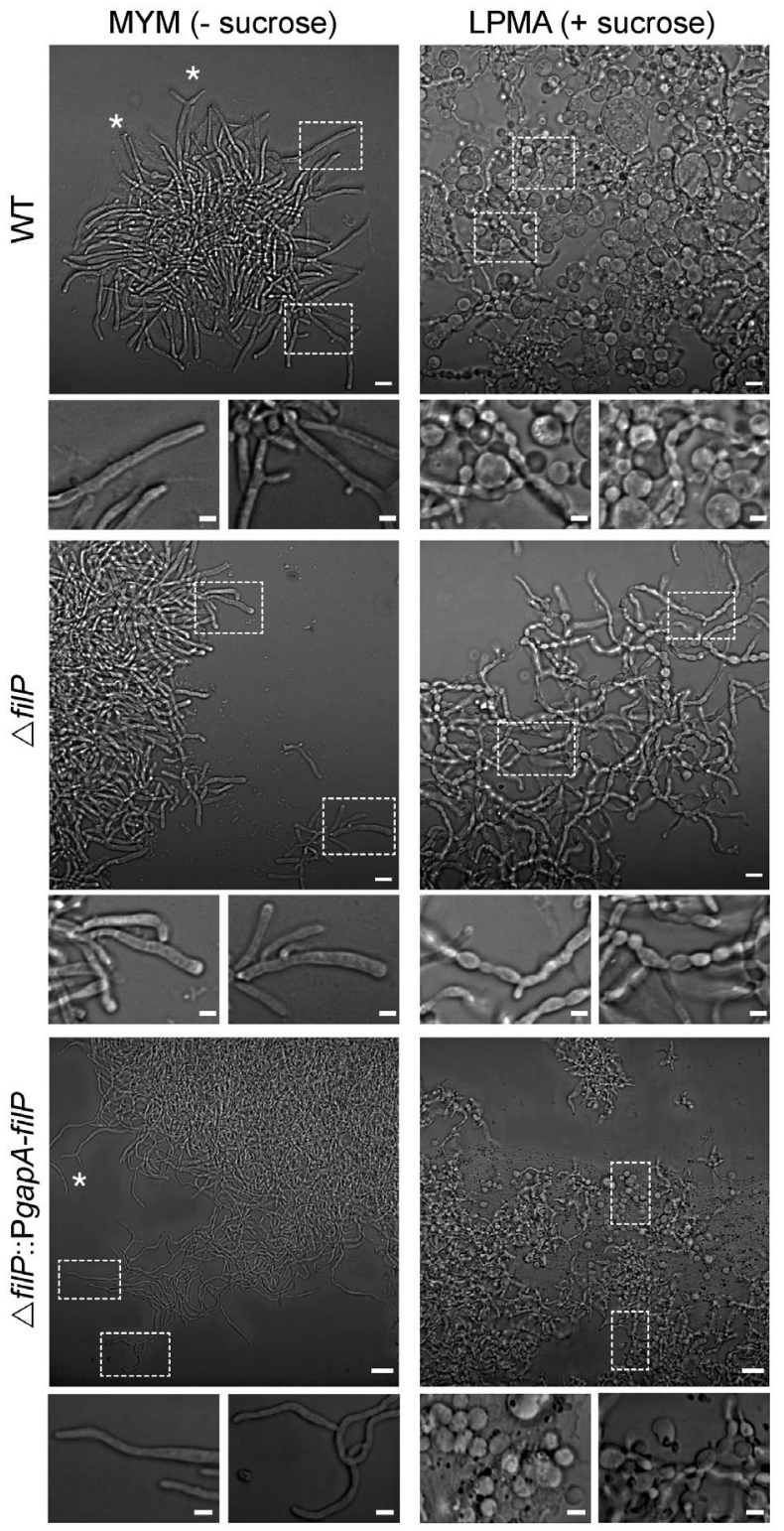

FIGURE 6 Morphology and S-cell extrusion of K. viridifaciens in micro-aerobic conditions. Micrographs of mycelia of the $K$. viridifaciens wild-type (top), $\Delta$ filP mutant (middle), and complemented mutant strain (bottom) cultured micro-aerobically on the solid medium MYM (no osmotic stress, left panels) or LPMA (high osmotic stress, right panels). Filaments of the wild-type strain and the complemented $\Delta$ filP mutant show tip splitting on MYM medium (asterisks). S-cells and vesicles are present in the wild-type and the complemented $\triangle$ filP mutant cultures on LPMA but are absent in the $\Delta$ filP mutant. The inlays show bulging hyphae in the LPMA-grown cultures. Scale bars represent $5 \mu \mathrm{m}$ or $2 \mu \mathrm{m}$ (inlays)

that in oxygen-limiting conditions, FilP is required for the extrusion of S-cells, as well as for the establishment of a normal mycelial architecture in the absence of osmotic stress.

\section{3 | DISCUSSION}

We previously showed that cell wall-deficient S-cells are formed by filamentous actinomycetes upon exposure to hyperosmotic stress, but the underlying regulation of this process was unknown. In this study, we reveal the detailed structural changes that accompany $\mathrm{S}$-cell formation and release for the first time. Furthermore, our results show that the absence of cytoskeletal protein FilP distorts hyphal morphology and blocks S-cell formation. S-cell formation appears to increase in the wild-type strain when grown in micro-aerobic conditions but remains absent in the strain lacking FilP. These results identify FilP as the first protein to be involved in the regulation of S-cell formation in $K$. viridifaciens. The increased formation of S-cells in oxygen-limiting conditions further suggests that $\mathrm{S}$-cells may serve an important function in oxygen-deprived environments.

Filamentous microorganisms incorporate new wall material at the hyphal tips. In such organisms, turgor pressure is important for growth and believed to physically stretch the wall to allow incorporation of cell wall precursors (Harold, 2002; Koch and Doyle, 1985; Lew, 2011; Steinberg, 2007). Hyperosmotic environments lower the turgor pressure and could, therefore, reduce the speed of apical wall synthesis. Additionally, this could also induce plasmolysis through the separation of the cytoplasmic membrane from the bacterial cell wall (Korber et al., 1996; Pilizota and Shaevitz, 2013; Rojas and Huang, 2018). Both events could contribute to S-cell formation and their release from the tip. Potentially, if cell wall synthesis but not membrane synthesis is affected by changes in turgor pressure, this could lead to a disbalance and relative overproduction of membrane.

Our cryoET data shows that the cell wall is thinner when $\mathrm{K}$. viridifaciens is exposed to hyperosmotic stress. Indeed, this would be expected given that osmotic upshifts lead to a growth arrest at the leading hyphal tip (Fuchino et al., 2017). As a consequence, less nascent PG is inserted and might result in a thinner and structurally weaker cell wall. As nascent PG is exclusively incorporated at the hyphal tip, this forms the weakest spot of the hyphal cell wall (Bibb et al., 2000) and explains why S-cells are extruded at the tip. The extrusion furthermore appears to depend on FilP, which assembles into a cytoskeletal network in hyphae undergoing active tip growth (Fuchino et al., 2013). FilP is thought to provide additional strength to these regions and fulfills a structural role in tip rigidity and elasticity. Indeed, previous work in S. coelicolor demonstrated that the hyphae of a constructed filP mutant were softer than those of the wild type (Bagchi et al., 2008). Hyphal rigidity is mainly determined by turgor pressure and the cell wall. At this moment we cannot differentiate between these factors in explaining why the extrusion of S-cells is abolished in the absence of FilP. Perhaps the necessary turgor pressure required for the extrusion of S-cells becomes too low in the filP mutant in hyperosmotic conditions. Alternatively, FilP could play an important, yet, nonessential role in cell wall synthesis or maturation, which is important for the extrusion of S-cells.

Besides being unable to extrude S-cells, the filP mutant of $K$. viridifaciens is strikingly similar to that of $S$. coelicolor. In both mutant strains, the mycelial morphology was affected, and the radial expansion of the colonies was diminished irrespective of the presence of high levels of osmolytes in the medium. This similarity is not surprising given that both bacteria use tip growth, and the involved machineries are conserved. How FilP mechanistically regulates mycelial morphology is an important direction for future work. 


\section{1 | A role for oxygen deprivation in S-cell extrusion}

Aerobic bacteria use oxygen as an electron acceptor in the electron transfer chain (ETC), which causes an increase in the levels of reactive oxygen species (ROS). Increased ROS levels appear to be an indirect result of blocking PG synthesis, leading to an excess of sugars metabolized via the TCA cycle and consequently more ROS generated as byproduct in the ETC. Notably, L-form bacteria were found to be sensitive to oxidative damage caused by high ROS levels (Kawai et al., 2015). Counteracting these high amounts of cytotoxic ROS compounds, as by redirecting carbon metabolism, allows the CWD cells to become robust and proliferating L-forms (Kawai et al., 2019; Kawai et al., 2015). When grown anaerobically, oxygen is not available as electron acceptor in the ETC chain, and therefore, ROS levels are lower. We here observed that $\mathrm{S}$-cells appear more abundant when $K$. viridifaciens is grown in microaerobic conditions. Whether the reduced oxygen levels facilitate the extrusion process or even lead to proliferation of S-cells is unknown.

Furthermore, the absence of FilP has a dramatic effect on the ability to form S-cells in oxygen-limiting conditions. To our knowledge, no direct link between the apical growth machinery and oxygen stress has been described before. The deviant tip morphology of the wild-type strain on growth medium with limited oxygen supports the hypothesis that oxygen levels influence the TIPOC. However, limited knowledge is available on micro-aerobic growth in filamentous actinomycetes, which may in fact be a condition that these bacteria frequently encounter in the heterogeneous soil environment.

As soil-dwelling bacteria, filamentous actinomycetes are often found in association with eukaryotic hosts, including plants (van der Meij et al., 2018). The root systems of plants are highly dynamic in their oxygen availability and can, depending on their abiotic influences, transition to an anoxic state (Larsen et al., 2015). Interestingly, CWD cells or L-forms can sustain in and traverse through plants. It has been hypothesized that the transition from intercellular to intracellular invasion leads to a loss of the cell wall in root tissue (Verma et al., 2018; Verma and White, 2018). Strawberry plants inoculated with B. subtilis L-forms showed that these bacterial cells can travel from the injection spot to up to $42 \mathrm{~cm}$ through the plant (Ferguson et al., 2000), which is likely facilitated by their smaller size and flexibility, allowing them to cross spaces that are inaccessible to walled cells. Likewise, we expect that CWD cells of filamentous bacteria such as $K$. viridifaciens or Streptomyces species would benefit from a transient wall-deficient state to maneuver through plants. Here, we show that micro-aerobic conditions promote $\mathrm{S}$-cell formation, an ability that could be encountered the anoxic environment of plant tissue. These results thus provide a lead in understanding where the formation of S-cells in nature is important.

\section{4 | EXPERIMENTAL PROCEDURES}

\subsection{Strains and media}

The wild-type strain Kitasatospora viridifaciens DSM40239 (Ramijan et al., 2017) was obtained from DSMZ. Spores were harvested after
7 days of growth on MYM agar medium (Stuttard, 1982). Vegetative mycelium was grown in the liquid medium TSBS (BD ${ }^{\mathrm{TM}}$ Tryptic Soy Broth supplemented with $10 \%(\mathrm{w} / \mathrm{v}$ ) sucrose). Liquid-grown cultures were incubated at $30^{\circ} \mathrm{C}$, in $100 \mathrm{ml}$ flasks equipped with coils while shaking at $200 \mathrm{rpm}$. To promote the extrusion of S-cells, high osmotic L-phase broth (LPB) or the solid L-phase medium (LPMA) were used (Ramijan et al., 2018). All cultures were incubated at $30^{\circ} \mathrm{C}$ and liquid cultures were grown in $100 \mathrm{ml}$ flasks without coil and gently shaken at $100 \mathrm{rpm}$.

\subsection{Growth of micro-aerobic cultures}

Spores or germinated spores of $K$. viridifaciens strains were inoculated on solid LPMA or MYM medium, after which the plates were placed inside a $2.5 \mathrm{~L}$ sealed jar (BBL ${ }^{\mathrm{TM}} \mathrm{GasPak}^{\mathrm{TM}}$ from BD Biosciences or from Merck) under micro-aerobic conditions created by using an oxygen-absorbing AnaeroGen sachet (Oxoid). The micro-aerobic atmosphere was monitored using methylene blue indicator strips. The jar was placed in an incubator set at $30^{\circ} \mathrm{C}$ for 2 weeks before the strains were further investigated.

\subsection{Construction of the $\Delta$ filP mutant}

The K. viridifaciens DSM40239 homolog of filP (BOQ63_RS19300) was identified by BLAST analysis using the FilP amino acid sequence of Streptomyces coelicolor (SCO5396). To create a $\Delta$ filP mutant, the filP gene was replaced with an apramycin-loxP cassette (aac(3)IV) via the unstable shuttle vector pWHM3-oriT (Światek et al., 2013; Vara et al., 1989) containing the flanking regions of the BOQ63_RS19300 gene (Supplementary Figure 1a). The flanking regions ranged from -780 bp to +11 upstream and from +916 to $+2,278$ downstream relative to the start codon of the filP gene. The PCR-amplified region of the upstream flank of filP was cut with restriction enzymes EcoRI and Xbal, whereas the downstream flank of filP was cut with Xbal and HindIII, to enable ligation into vector pWHM3-oriT cut with EcoRI and HindIII. The apramycin cassette was inserted in between the filP flanks via an Xbal restriction digest and ligation, resulting in vector $\mathrm{pWHM} 3$-oriT-filP. This vector was verified using a restriction digest with combinations of the abovementioned restriction enzymes (see Supplementary Figure 1c). The vector was introduced into the $K$. viridifaciens wild type via conjugation with $E$. coli ET12567/pUZ8002 (Kieser et al., 2000), followed by selection for exconjugants that were apramycin resistance and thiostrepton sensitive. The successful deletion of the filP gene was verified by PCR (Supplementary Figure 1d). Mutant M1 was chosen and further used in this study.

\subsection{Complementation of the $\Delta$ filP mutant}

The gapA promoter of sco1947 and coding sequence of filP (BOQ63_RS19300) were amplified from genomic DNA of $S$. 
coelicolor and K. viridifaciens DSM40239 using primers gapA-F (CGCGGATCC GTCCTCGCCGACGAGGCCTC)/gapA-R (GGGAATT CCATATG GAACCGATCTCCTCGTTGGTAC) and filp-F (GGAATTCC ATATGAGCGACAGTCACTCCCCTCACGGCTTCG)/filp-R (TGCTCTAGATCAGCGCGACTGCTGGGCGGGCAC), respectively. The amplified products were then ligated together in pIJ82 using the restriction enzymes BamHI-Ndel (gapA) and Ndel-Xbal (filP), yielding the PgapA:filP plasmid. Finally, the constructed plasmid was introduced into the $\triangle$ filP mutant of $K$. viridifaciens via conjugation (Kieser et al., 2000).

\section{5 | Fluorescence and time-lapse microscopy}

To stain nucleic acids, SYTO9 (Molecular Probes ${ }^{\mathrm{TM}}$ ) was added to samples obtained from liquid LPB or TSBS broth to a final concentration of $0.5 \mu \mathrm{M}$. To fluorescently label lipids, FM5-95 (Molecular Probes $^{\mathrm{TM}}$, Invitrogen) or SynapseRed C2M (PromoKine, PromoCell $\mathrm{GmbH}$ ) was used to a final concentration of $0.02 \mu \mathrm{g} / \mathrm{ml}$. The fluorescently stained cells were placed in a $35 \mathrm{~mm}$ imaging $\mu$-dish (Ibidi ${ }^{\circledR}$ ) and covered by an agar pad. The samples were analyzed using an inverted Zeiss Axio Observer Z1 confocal microscope equipped with an incubation chamber and Temperature Module S (PECON) stagetop set to $30^{\circ} \mathrm{C}$ for time-lapse recordings. Fluorescent microscopy images were collected using Zen software (Zeiss) and were viewed, analyzed, and edited using ImageJ/FIJI (Schindelin et al., 2012).

\subsection{Cryo-electron microscopy and tomography}

$K$. viridifaciens was cultured in liquid LPB medium and after 6 (cryoET) or $16 \mathrm{hr}$ (cryo-TEM) 3,5 $\mu$ of the culture was mixed with $10 \mathrm{~nm}$ gold fiducial markers (Cell Microscopy Core, UMC Utrecht) and applied onto glow-discharged R2/2 200 mesh holey carbon EM grids (Quantifoil). The grid was then rapidly plunged in liquid ethane using the Leica GP automated freeze-plunger, generating a vitrified sample. The vitrified samples were observed using a 626 side-entry cryo-holder (Gatan) and a $120 \mathrm{kV}$ Talos cryo-TEM (Thermo Fisher Scientific) equipped with a Lab6 electron source and Ceta detector. Cryo-TEM tilt series for tomography were collected at the Netherlands Center for Nanoscopy (NeCEN, Leiden) using a $300 \mathrm{kV}$ Titan Krios cryo-TEM (Thermo Fisher Scientific) equipped with a FEG electron gun, GIF energy filter, and K2 direct electron detector (Gatan). Tilt series were collected ranging from -60 to +60 with an increment of 2 degrees, and a pixel size of $4.241 \mathrm{~A}$. The data were collected using UCSF software (Zheng et al., 2004) and reconstructed to tomograms using IMOD software (Kremer et al., 1996).

\section{7 | Data analysis}

Cryo-TEM data were used to determine the cell wall thickness of $K$. viridifaciens hyphae grown under high osmotic stress (LPB medium,
$20 \%$ sucrose) and to compare with hyphae grown under non-stressed conditions (LPB, 0\% sucrose). Of both growth conditions, 15 hyphal tips were imaged. Per tip, three measurements of the cell wall thickness were obtained using ImageJ/FIJI (Schindelin et al., 2012).

\section{ACKNOWLEDGMENTS}

We would like to thank Helga van der Heul (Institute of Biology, Leiden University, the Netherlands) for providing the pWHM3-oriT vector. The cryo-ET work in this study has been supported by iNEXT, PID:2265, funded by the Horizon2020 programme of the European Commission. Work in the Claessen lab is funded by a Vici grant from NWO (VI.C.192.002).

\section{CONFLICT OF INTEREST}

There are no apparent conflicts of interest.

\section{AUTHOR CONTRIBUTIONS}

Ultee, Zhong, and Shitut collected the data and aided in data analysis. Ultee, Briegel, and Claessen designed the experiments. Ultee, Briegel, and Claessen wrote the paper with input from all coauthors.

\section{DATA AVAILABILITY STATEMENT}

The data that support the findings of this study are available from the corresponding author upon reasonable request.

\section{ORCID}

Ariane Briegel iD https://orcid.org/0000-0003-3733-3725

Dennis Claessen (iD https://orcid.org/0000-0002-0789-2633

\section{REFERENCES}

Allan, E.J., Hoischen, C. and Gumpert, J. (2009) Bacterial L-forms. Advances in Applied Microbiology, 68, 1-39.

Bagchi, S., Tomenius, H., Belova, L.M. and Ausmees, N. (2008) Intermediate filament-like proteins in bacteria and a cytoskeletal function in Streptomyces. Molecular Microbiology, 70, 1037-1050.

Bibb, M.J., Molle, V. and Buttner, M.J. (2000) $\sigma(B l d N)$, an extracytoplasmic function RNA polymerase sigma factor required for aerial mycelium formation in Streptomyces coelicolor A3(2). Journal of Bacteriology, 182, 4606-4616.

Claessen, D. and Errington, J. (2019) Cell wall-deficiency as a coping strategy for stress. Trends in Microbiology, 27, 1025-1033.

Dörr, T., Alvarez, L., Delgado, F., Davis, B.M., Cava, F. and Waldor, M.K. (2016) A cell wall damage response mediated by a sensor kinase/response regulator pair enables beta-lactam tolerance. Proceedings of the National Academy of Sciences, 113, 404-409.

Ferguson, C.M.J., Booth, N.A. and Allan, E.J. (2000) An ELISA for the detection of Bacillus subtilis L-form bacteria confirms their symbiosis in strawberry. Letters in Applied Microbiology, 31, 390-394.

Flärdh, K. (2003) Essential role of DivIVA in polar growth and morphogenesis in Streptomyces coelicolor A3(2). Molecular Microbiology, 49, 1523-1536.

Flärdh, K., Richards, D.M., Hempel, A.M., Howard, M. and Buttner, M.J. (2012) Regulation of apical growth and hyphal branching in Streptomyces. Current Opinion in Microbiology, 15, 737-743.

Fuchino, K., Bagchi, S., Cantlay, S., Sandblad, L., Wu, D., Bergman, J. et al (2013) Dynamic gradients of an intermediate filament-like cytoskeleton are recruited by a polarity landmark during apical growth. Proceedings of the National Academy of Sciences, 110, E1889-E1897. 
Fuchino, K., Flärdh, K., Dyson, P. and Ausmees, N. (2017) Cell-biological studies of osmotic shock response in Streptomyces spp. Journal of Bacteriology, 199, e00465-e416.

Harold, F.M. (2002) Force and compliance: rethinking morphogenesis in walled cells. Fungal Genetics and Biology, 37, 271-282.

Hempel, A.M., Wang, S.B., Letek, M., Gil, J.A. and Flärdh, K. (2008) Assemblies of DivIVA mark sites for hyphal branching and can establish new zones of cell wall growth in Streptomyces coelicolor. Journal of Bacteriology, 190, 7579-7583.

Holmes, N.A., Walshaw, J., Leggett, R.M., Thibessard, A., Dalton, K.A., Gillespie, M.D. et al (2013) Coiled-coil protein Scy is a key component of a multiprotein assembly controlling polarized growth in Streptomyces. Proceedings of the National Academy of Sciences, 110, E397-E406.

Höltje, J.V. (1998) Growth of the stress-bearing and shape-maintaining murein sacculus of Escherichia coli. Microbiology and Molecular Biology Reviews, 62, 181-203.

Kawai, Y., Mercier, R., Mickiewicz, K., Serafini, A., Sório de Carvalho, L.P. and Errington, J. (2019) Crucial role for central carbon metabolism in the bacterial L-form switch and killing by beta-lactam antibiotics. Nature Microbiology, 4, 1716-1726.

Kawai, Y., Mercier, R., Wu, L.J., Domínguez-Cuevas, P., Oshima, T. and Errington, J. (2015) Cell growth of wall-free L-form bacteria is limited by oxidative damage. Current Biology, 25, 1613-1618.

Kawai, Y., Mickiewicz, K. and Errington, J. (2018) Lysozyme counteracts $\beta$-Lactam antibiotics by promoting the emergence of L-form bacteria. Cell, 172, 1038-1049.e1010.

Kieser, T., Bibb, M.J., Buttner, M.J., Chater, K.F. and Hopwood, D.A. (2000) Practical Streptomyces Genetics. Norwich: The John Innes Foundation.

Koch, A.L. and Doyle, R.J. (1985) Inside-to-outside growth and turnover of the wall of gram-positive rods. Journal of Theoretical Biology, 117, 137-157.

Kois-Ostrowska, A., Strzałka, A., Lipietta, N., Tilley, E., ZakrzewskaCzerwińska, J., Herron, P. et al (2016) Unique function of the bacterial chromosome segregation machinery in apically growing Streptomyces - targeting the chromosome to new hyphal tubes and its anchorage at the tips. PLoS Genetics, 12, e1006488.

Korber, D.R., Choi, A., Wolfaardt, G.M. and Caldwell, D.E. (1996) Bacterial plasmolysis as a physical indicator of viability. Applied and Environmental Microbiology, 62, 3939-3947.

Kremer, J.R., Mastronarde, D.N. and McIntosh, J.R. (1996) Computer visualization of three-dimensional image data using IMOD. Journal of Structural Biology, 116, 71-76.

Larsen, M., Santner, J., Oburger, E., Wenzel, W.W. and Glud, R.N. (2015) $\mathrm{O} 2$ dynamics in the rhizosphere of young rice plants (Oryza sativa L.) as studied by planar optodes. Plant and Soil, 390, 279-292.

Leaver, M., Dominguez-Cuevas, P., Coxhead, J.M., Daniel, R.A. and Errington, J. (2009) Life without a wall or division machine in Bacillus subtilis. Nature, 457, 849-853.

Lew, R.R. (2011) How does a hypha grow? The biophysics of pressurized growth in fungi. Nature Reviews: Microbiology, 9, 509-518.

Monahan, L.G., Turnbull, L., Osvath, S.R., Birch, D., Charles, I.G. and Whitchurch, C.B. (2014) Rapid conversion of Pseudomonas aeruginosa to a spherical cell morphotype facilitates tolerance to carbapenems and penicillins but increases susceptibility to antimicrobial peptides. Antimicrobial Agents \& Chemotherapy, 58, 1956-1962.

Pazos, M., Peters, K. and Vollmer, W. (2017) Robust peptidoglycan growth by dynamic and variable multi-protein complexes. Current Opinion in Microbiology, 36, 55-61.

Pilizota, T. and Shaevitz, J.W. (2013) Plasmolysis and cell shape depend on solute outer-membrane permeability during hyperosmotic shock in E. coli. Biophysical Journal, 104, 2733-2742.

Ramijan, K., Ultee, E., Willemse, J., Zhang, Z., Wondergem, J.A.J., van der Meij, A. (2018) Stress-induced formation of cell wall-deficient cells in filamentous actinomycetes. Nature Communications, 9, 5164.

Ramijan, K., van Wezel, G.P. and Claessen, D. (2017) Genome sequence of the filamentous actinomycete Kitasatospora viridifaciens. Genome Announc, 5, e01560-e1516.

Rojas, E.R. and Huang, K.C. (2018) Regulation of microbial growth by turgor pressure. Current Opinion in Microbiology, 42, 62-70.

Schindelin, J., Arganda-Carreras, I., Frise, E., Kaynig, V., Longair, M., Pietzsch, T. et al (2012) Fiji: an open-source platform for biological-image analysis. Nature Methods, 9, 676-682.

Steinberg, G. (2007) Hyphal growth: a tale of motors, lipids, and the Spitzenkörper. Eukaryotic Cell, 6, 351-360.

Stuttard, C. (1982) Temperate phages of Streptomyces venezuelae: lysogeny and host specificity shown by phages SV1 and SV2. Journal of General Microbiology, 128, 115-121.

Światek, M.A., Gubbens, J., Bucca, G., Song, E., Yang, Y.H., Laing, E. et al (2013) The ROK family regulator Rok7B7 pleiotropically affects xylose utilization, carbon catabolite repression, and antibiotic production in Streptomyces coelicolor. Journal of Bacteriology, 195, 1236-1248.

Typas, A., Banzhaf, M., Gross, C.A. and Vollmer, W. (2012) From the regulation of peptidoglycan synthesis to bacterial growth and morphology. Nature Reviews: Microbiology, 10, 123-136.

van der Meij, A., Willemse, J., Schneijderberg, M.A., Geurts, R., Raaijmakers, J.M. and van Wezel, G.P. (2018) Inter- and intracellular colonization of Arabidopsis roots by endophytic actinobacteria and the impact of plant hormones on their antimicrobial activity. Antonie van Leeuwenhoek, 111, 679-690.

van Teeffelen, S. and Renner, L.D. (2018) Recent advances in understanding how rod-like bacteria stably maintain their cell shapes. F1000Res, 7, 241.

Vara, J., Lewandowska-Skarbek, M., Wang, Y.G., Donadio, S. and Hutchinson, C.R. (1989) Cloning of genes governing the deoxysugar portion of the erythromycin biosynthesis pathway in Saccharopolyspora erythraea (Streptomyces erythreus). Journal of Bacteriology, 171, 5872-5881.

Verma, S.K., Kingsley, K., Bergen, M., English, C., Elmore, M., Kharwar, R.N. et al (2018) Bacterial endophytes from rice cut grass (Leersia oryzoides L.) increase growth, promote root gravitropic response, stimulate root hair formation, and protect rice seedlings from disease. Plant and Soil, 422, 223-238.

Verma, S.K. and White, J.F. (2018) Indigenous endophytic seed bacteria promote seedling development and defend against fungal disease in browntop millet (Urochloa ramosa L.). Journal of Applied Microbiology, 124, 764-778.

Zheng, Q.S., Braunfeld, M.B., Sedat, J.W. and Agard, D.A. (2004) An improved strategy for automated electron microscopic tomography. Journal of Structural Biology, 147, 91-101.

\section{SUPPORTING INFORMATION}

Additional supporting information may be found online in the Supporting Information section.

How to cite this article: Ultee E, Zhong X, Shitut S, Briegel A, Claessen D. Formation of wall-less cells in Kitasatospora viridifaciens requires cytoskeletal protein FilP in oxygenlimiting conditions. Mol Microbiol. 2020;00:1-10. https://doi. org/10.1111/mmi.14662 